\title{
Diffusion and Deterministic Systems
}

\author{
M. Tyran-Kamińska * \\ Institute of Mathematics, University of Silesia, Bankowa 14, 40-007 Katowice, Poland
}

\begin{abstract}
We show that simple diffusion processes are weak limits of piecewise continuous processes constructed within a totally deterministic framework. The proofs are based on the continuous mapping theorem and the functional central limit theorem.
\end{abstract}

Keywords and phrases: functional limit theorem, piecewise monotonic maps, OrnsteinUhlenbeck process, Langevin equation, diffusion

Mathematics Subject Classification: 28D05, 37A50, 60F17, 60G51, 60J60

\section{Introduction}

Trajectories of discrete time dynamical systems can be unpredictable and behave in a random-like way. A series of numerical experiments with Langevin-type equations reported in $[1,2]$ led to speculate that one can observe diffusive behaviour. In [14] we have reviewed how the Wiener process can be reproduced by purely deterministic systems and studied a one dimensional Langevin-type equation

$$
\frac{d v(t)}{d t}=-\gamma v(t)+\eta(t)
$$

Here $\gamma$ is a positive constant and a perturbation $\eta(t)$ consists of a series of delta-function like impulses given by

$$
\eta(t)=\kappa(\tau) \sum_{j=0}^{\infty} h(\xi(t)) \delta(t-j \tau)
$$

with $\tau>0$ and a scaling parameter $\kappa(\tau)>0$. The real valued function $h$ is defined on a probability space $(Y, \mathcal{B}, \nu)$ (a measure space with $\nu(Y)=1)$ and $\xi(t)=\xi_{j}$ for $j \tau \leq t<(j+1) \tau, j \geq 0$, with $\xi_{j+1}=T\left(\xi_{j}\right)$ and $\xi_{0}(y)=y, y \in Y$, where $T: Y \rightarrow Y$ is a 'chaotic' map. One particular example is given by the tent map $T$ on the interval $[-1,1]$ defined by:

$$
T(y)= \begin{cases}2\left(y+\frac{1}{2}\right) & \text { for } \quad y \in[-1,0) \\ 2\left(\frac{1}{2}-y\right) & \text { for } \quad y \in[0,1]\end{cases}
$$

\footnotetext{
${ }^{*}$ Corresponding author. E-mail: mtyran@us.edu.pl
} 
and $\nu$ being the normalized Lebesgue measure on $[-1,1]$. For any $\tau>0$ we say that $v_{\tau}(t), t \geq 0$, is a solution of (1.1) starting at $v_{0} \in \mathbb{R}$ if, for each $j \geq 0, v_{\tau}(t)$ is a solution of problem

$$
\left\{\begin{array}{l}
\frac{d v_{\tau}(t)}{d t}=-\gamma v_{\tau}(t), \quad t \in(j \tau,(j+1) \tau), \\
v_{\tau}(j \tau)=v_{\tau}(j \tau-)+\kappa(\tau) h\left(\xi_{j}\right),
\end{array}\right.
$$

where we set $v_{\tau}(0-)=v_{0}$ and we write $v_{\tau}(s-)$ for the left-hand $\operatorname{limit} \lim _{t \uparrow s} v_{\tau}(t)$ when $s>0$. The solution is of the form

$$
v_{\tau}(t)=e^{-\gamma t} v_{0}+\kappa(\tau) e^{-\gamma t} \sum_{j \tau \leq t} e^{\gamma \tau j} h\left(\xi_{j}\right), \quad t \geq 0 .
$$

It was shown in [14, Section 4] that in the limit $\tau \rightarrow 0$ we may reproduce the characteristics of an Ornstein-Uhlenbeck velocity process $V=\{V(t): t \geq 0\}$ given by

$$
V(t)=e^{-\gamma t} v_{0}+e^{-\gamma t} \int_{0}^{t} e^{\gamma s} \sigma d W(s), \quad t \geq 0,
$$

where $W=\{W(t): t \geq 0\}$ is a Wiener process, provided that $\kappa(\tau)^{2} / \tau$ converges to 1 as $\tau \rightarrow 0$ and the functional central limit theorem (FCLT) holds for $h$

$$
w_{\tau} \stackrel{d}{\rightarrow} \sigma W, \quad \text { where } \quad w_{\tau}(t)=\sqrt{\tau} \sum_{j \tau \leq t} h\left(\xi_{j}\right), \quad t \geq 0 .
$$

The notation ' $\stackrel{d}{\rightarrow}$ ' refers to weak convergence of distributions of stochastic processes with paths in the space $\mathbb{D}$ of càdlàg functions, i.e., functions $\psi$ on $[0, \infty)$ that are right-continuous and have finite left-hand limits $\psi(t-)$ for every $t>0$, endowed with the topology of uniform convergence on compact subsets of $[0, \infty)$; see $[3,12,24]$ for weak convergence of probability measures and stochastic-process limits. We recall background material on convergence to processes with continuous sample paths in section 2 .

The first general FCLT was obtained by Donsker [5] for random walks, which in our notation corresponds to the random variables $h\left(\xi_{j}\right)$ being independent and identically distributed (i.i.d.) with mean 0 and variance $\sigma^{2}$. It follows from the central limit theorem for random walks and it was subsequently generalized from random walks to dependent random variables; see $[3,14,16]$ and the references therein. In section 3 we recall a sufficient condition for FCLT from [20] and a couple of examples from [14].

Since the paths of $w_{\tau}$ are piecewise constant functions with finitely many discontinuities on every time interval $[0, t]$, we can write

$$
w_{\tau}(t)=\sum_{0 \leq s \leq t} \Delta w_{\tau}(s),
$$

where we set $w_{\tau}(0-)=0$ and $\Delta w_{\tau}(s)=w_{\tau}(s)-w_{\tau}(s-), s \geq 0$. Consequently, if $\phi$ is a locally bounded Borel measurable function on $\mathbb{R}_{+}$then the Lebesgue-Stieltjes integral of $\phi$ with respect to $w_{\tau}$ over the interval $[0, t]$ is equal to

$$
\int_{0}^{t} \phi(s) d w_{\tau}(s)=\sum_{0 \leq s \leq t} \phi(s) \Delta w_{\tau}(s) .
$$

For the particular representation in (1.6) we have

$$
\int_{0}^{t} \phi(s) d w_{\tau}(s)=\sqrt{\tau} \sum_{0 \leq j \tau \leq t} \phi(j \tau) h\left(\xi_{j}\right) .
$$

Thus, the solution (1.4) with $\kappa(\tau)=\sqrt{\tau}$ has the representation

$$
v_{\tau}(t)=e^{-\gamma t} v_{0}+e^{-\gamma t} \int_{0}^{t} e^{\gamma s} d w_{\tau}(s), \quad t \geq 0 .
$$


We can also rewrite (1.1) as the integral equation

$$
v_{\tau}(t)=v_{0}-\gamma \int_{0}^{t} v_{\tau}(s) d s+\int_{0}^{t} d w_{\tau}(s), \quad t \geq 0,
$$

and use the shorthand notation in the differential form

$$
d v_{\tau}(t)=-\gamma v_{\tau}(t) d t+d w_{\tau}(t)
$$

It is our aim to consider the general Langevin-type equation, as studied numerically in [2],

$$
d z_{\tau}(t)=A\left(z_{\tau}(t)\right) d t+d w_{\tau}(t)
$$

and to give a simple proof of the following result.

Theorem 1.1. Let $z_{\tau}$ be the unique solution of

$$
z_{\tau}(t)=z_{0}+\int_{0}^{t} A\left(z_{\tau}(s)\right) d s+w_{\tau}(t), \quad t \geq 0
$$

for all sufficiently small $\tau$, where $A: \mathbb{R} \rightarrow \mathbb{R}$ is of class $C^{1}$. If $w_{\tau} \stackrel{d}{\rightarrow} \sigma W$ as $\tau \rightarrow 0$ then $z_{\tau} \stackrel{d}{\rightarrow} Z$ where $Z$ is the solution of

$$
Z(t)=z_{0}+\int_{0}^{t} A(Z(s)) d s+\sigma W(t),
$$

provided that $Z$ is non-exploding, i.e., the explosion time $e=\inf \{t \geq 0:|Z(t)|=+\infty\}$ is infinite with probability one.

Weak convergence of distributions of stochastic processes is often obtained by showing that the family is weakly relatively compact and all its limit points are the same, see $[7,19]$ for convergence in the class of Markov processes and [12] in the case of general semimartingales and processes derived from them. We aim to make our results more accessible by using a direct approach through the continuous mapping theorem (CMT), recalled in section 2, which allows us to obtain new limit theorems from existing ones. To use the CMT we need measurability and continuity of the corresponding mapping which is proved in Theorem 4.2 and generalizes [17, Theorem 4.1].

We also extend our approach to include multiplicative perturbations. We add a perturbation $\eta$ as in (1.2) to the equation of the form

$$
\frac{1}{x(t)} \frac{d x(t)}{d t}=g(x(t))
$$

To simplify our exposition we assume that $h$ as in (1.2) is bounded, that $g:(0, \infty) \rightarrow \mathbb{R}$ in $(1.10)$ is of class $C^{1}$, and that for each $x_{0} \in(0, \infty)$, the solution $x$ of (1.10) satisfying $x(0)=x_{0}$ is defined for all times $t \geq 0$, so that it does not exit the state space $(0, \infty)$ in a finite time. Thus for each $\tau>0$, we consider the equation

$$
x_{\tau}(t)=x_{0}+\int_{0}^{t} x_{\tau}(s) g\left(x_{\tau}(s)\right) d s+\int_{0}^{t} x_{\tau}(s-) d w_{\tau}(s), \quad t \geq 0,
$$

with $x_{0} \in(0, \infty)$, where both integrals in (1.11) are Lebesgue-Stieltjes integrals. Since the solution of (1.11) is continuous on intervals of the form $(j \tau,(j+1) \tau)$, we can replace $x(s)$ with $x(s-)$ in the first integral, which leads to the following differential form

$$
d x_{\tau}(t)=x_{\tau}(t-) g\left(x_{\tau}(t-)\right) d t+x_{\tau}(t-) d w_{\tau}(t) .
$$


Theorem 1.2. Suppose that the FCLT (1.6) holds where $h$ and $\xi_{j}$ are such that for all $t \geq 0$

$$
\tau \sum_{j \tau \leq t} h^{2}\left(\xi_{j}\right) \rightarrow \sigma_{0}^{2} t \quad \text { a.s. as } \tau \rightarrow 0 .
$$

Let $x_{\tau}$ be the solution of (1.11). Then $x_{\tau} \stackrel{d}{\rightarrow} X$ as $\tau \rightarrow 0$ where $X$ is the solution of the Itô equation

$$
X(t)=x_{0}+\int_{0}^{t} X(s)\left(g(X(s))+\frac{1}{2}\left(\sigma^{2}-\sigma_{0}^{2}\right)\right) d s+\sigma \int_{0}^{t} X(s) d W(s),
$$

provided that $X$ is non-exploding, i.e., the explosion time $\zeta=\inf \{t: X(t) \notin(0, \infty)\}$ is infinite with probability one.

We provide some examples of deterministic perturbations in section 3. The case of $\sigma^{2} \neq \sigma_{0}^{2}$ is possible only when the random variables $h\left(\xi_{j}\right)$ are dependent, since for i.i.d. sequences condition (1.12) holds with $\sigma_{0}^{2}=\sigma^{2}$ by the law of large numbers.

Corollary 1.3. Suppose that $h\left(\xi_{j}\right), j \geq 0$, are independent and identically distributed random variables with mean zero and variance $\sigma^{2}>0$. Let $x_{\tau}$ be the solution of (1.11). Then $x_{\tau} \stackrel{d}{\rightarrow} X$ as $\tau \rightarrow 0$ where $X$ is the solution of the Itô equation

$$
X(t)=x_{0}+\int_{0}^{t} X(s) g(X(s)) d s+\sigma \int_{0}^{t} X(s) d W(s),
$$

provided that $X$ is non-exploding.

Remark 1.4. If instead of $w_{\tau}$ we use its linearly interpolated continuous version

$$
\tilde{w}_{\tau}(t)=w_{\tau}(j \tau)+\frac{t-j \tau}{\sqrt{\tau}} h\left(\xi_{j+1}\right), \quad j \tau \leq t<(j+1) \tau, j=0,1, \ldots,
$$

then the solution of the equation

$$
\tilde{x}_{\tau}(t)=x_{0}+\int_{0}^{t} \tilde{x}_{\tau}(s) g\left(\tilde{x}_{\tau}(s)\right) d s+\int_{0}^{t} \tilde{x}_{\tau}(s) d \tilde{w}_{\tau}(s), \quad t \geq 0,
$$

will converge in distribution to the process

$$
\tilde{X}(t)=x_{0}+\int_{0}^{t} \tilde{X}(s) g(\tilde{X}(s)) d s+\sigma \int_{0}^{t} \tilde{X}(s) \circ d W(s)
$$

where the last integral is the Stratonovich stochastic integral. This is the Wong-Zakai approximation [26]. Then the corresponding Itô equation for $\tilde{X}$ is of the form

$$
\tilde{X}(t)=x_{0}+\int_{0}^{t}\left(\tilde{X}(s) g(\tilde{X}(s))+\frac{\sigma^{2}}{2} \tilde{X}(s)\right) d s+\sigma \int_{0}^{t} \tilde{X}(s) d W(s)
$$

and our approximation gives a different process in the limit.

The Wong-Zakai approximations and examples where in the limit one obtains neither Itô nor Stratonovich equations are described in [11]. Finally, we refer the reader to $[9,10,15]$ and the references therein for related results. In particular, $[9,10]$ studied linearly interpolated discrete time sequences with perturbations coming from ergodic systems extending in yet another direction the approach of $[1,2]$. A description of possible generalizations of our results is provided in the last section. 


\section{Preliminaries}

In this section we collect basic tools and notions used in the paper. Recall that $W=\{W(t): t \geq 0\}$ defined on a probability space $(\Omega, \mathcal{F}, \mathbb{P})$ is called a Wiener process (standard Brownian motion) if:

1. The increments are independent: for all times $0 \leq t_{0}<t_{1}<\ldots<t_{n}$

$$
\mathbb{P}\left(W\left(t_{i}\right)-W\left(t_{i-1}\right) \in B_{i}, i \leq n\right)=\prod_{i \leq n} \mathbb{P}\left(W\left(t_{i}\right)-W\left(t_{i-1}\right) \in B_{i}\right)
$$

and all Borel measurable sets $B_{1}, \ldots, B_{n} \in \mathcal{B}(\mathbb{R})$.

2. For $t>0, s \geq 0, W(t+s)-W(s)$ is Gaussian distributed with mean 0 and variance $t$.

3. The sample paths are continuous and $W(0)=0$.

The existence of Brownian motion was shown by Wiener [25], who constructed the corresponding distribution of $W$ as a probability measure $\mathbb{P}$ on $\Omega=C[0, \infty)$, the space of continuous functions $[0, \infty)$, under which the coordinate process $W(t)=\pi_{t}, t \geq 0$, has the required properties, where each $\pi_{t}: C[0, \infty) \rightarrow \mathbb{R}$ denotes the projection $\pi_{t}(\omega)=\omega(t), \omega \in C[0, \infty)$. The space $C[0, \infty)$ endowed with the topology of uniform convergence on compact subsets of $[0, \infty)$ is metrizable with a complete and separable metric $d_{u}$. The Borel $\sigma$-algebra of subsets of $C[0, \infty)$, i.e., the smallest $\sigma$-algebra containing all open sets, is the same as the $\sigma$-algebra generated by projections, i.e., the smallest $\sigma$-algebra with respect to which the projections $\pi_{t}, t \geq 0$, are measurable. Thus finite-dimensional distributions uniquely determine the measure $\mathbb{P}$.

We endow $\mathbb{D}$ with the local uniform topology and we write $\psi_{n} \rightarrow \psi$ iff

$$
\left\|\psi_{n}-\psi\right\|_{m}=\sup _{0 \leq s \leq m}\left|\psi_{n}(s)-\psi(s)\right| \rightarrow 0 \quad \text { for all } m>0 .
$$

This topology in $\mathbb{D}$ is metrizable with a complete metric $d_{u}$, but it fails to be separable and its Borel $\sigma$ algebra contains too many sets, as concern the weak convergence of distributions of stochastic processes. Thus, we consider $\mathbb{D}$ with the $\sigma$-algebra $\mathcal{D}$ generated by projections $\pi_{t}: \mathbb{D} \rightarrow \mathbb{R}, t \geq 0$, which is the same as the $\sigma$-algebra generated by open balls in $\left(\mathbb{D}, d_{u}\right)[3$, Section 15$]$.

We write $W_{\tau} \stackrel{d}{\rightarrow} W_{0}$ as $\tau \rightarrow 0$ iff for all $\mathcal{D}$-measurable bounded continuous $f: \mathbb{D} \rightarrow \mathbb{R}$

$$
E_{\nu} f\left(W_{\tau}\right) \underset{\tau \rightarrow 0}{\longrightarrow} \operatorname{Ef}\left(W_{0}\right)
$$

where $E_{\nu}, E$ are the expectation operators, $E_{\nu}$ on the probability space $(Y, \mathcal{B}, \nu)$ and $E$ on a probability space $(\Omega, \mathcal{F}, \mathbb{P})$ on which the process $W_{0}$ is defined. We note that the space $\mathbb{D}$ is more naturally endowed with the Skorohod $J_{1}$ topology [18], which is metrizable with a complete and separable metric $d$ and the Borel $\sigma$-algebra of $(\mathbb{D}, d)$ is then equal to $\mathcal{D}$ (see e.g. [23], [3, Section 16] or [12, Section 6]). In particular, a function $f: \mathbb{D} \rightarrow \mathbb{R}$ which is continuous on $(\mathbb{D}, d)$ is $\mathcal{D}$-measurable, since for each $a$ the set $\{\psi \in \mathbb{D}: f(\psi)<a\}$ is open in $(\mathbb{D}, d)$, thus belongs to $\mathcal{D}$, but a continuous function on $\left(\mathbb{D}, d_{u}\right)$ need not be $\mathcal{D}$-measurable. Since we consider convergence to processes with continuous sample paths and $C[0, \infty)$ is a closed and separable subset of $\left(\mathbb{D}, d_{u}\right)$, it follows from [3, Theorem 6.6] that $W_{\tau} \stackrel{d}{\rightarrow} W_{0}$ is equivalent to the weak convergence of distributions in $\mathbb{D}$ with the Skorohod $J_{1}$-topology.

We say that a mapping $G$ on $\mathbb{D}$ is a.s. continuous at $W_{0}$, if there is a set $\mathbb{D}_{0} \in \mathcal{D}$ such that $\mathbb{P}\left(W_{0} \in\right.$ $\left.\mathbb{D}_{0}\right)=1$ and $G\left(\psi_{n}\right) \rightarrow G(\psi)$ whenever $\psi_{n} \rightarrow \psi$ with $\psi \in \mathbb{D}_{0}$. We need the following version of the continuous mapping theorem (see [3, Theorem 6.3]).

Theorem 2.1 (CMT). Let $G: \mathbb{D} \rightarrow \mathbb{D}$ be a measurable mapping. Suppose that $W_{\tau} \stackrel{d}{\rightarrow} W_{0}$ as $\tau \rightarrow 0$ and $W_{0}$ has continuous sample paths. If $G$ is a.s. continuous at $W_{0}$ then $G\left(W_{\tau}\right) \stackrel{d}{\rightarrow} G\left(W_{0}\right)$. 
Observe that a mapping $g: \mathbb{D} \rightarrow \mathbb{D}$ is measurable iff $\pi_{t} \circ G: \mathbb{D} \rightarrow \mathbb{R}$, where $\pi_{t}$ is the projection, is $\mathcal{D}$-measurable for all $t \geq 0$. Finally, if the FCLT (1.6) holds then for every $\mathcal{D}$-measurable $f: \mathbb{D} \rightarrow \mathbb{R}$ which is a.s. continuous at $W$

$$
f\left(w_{\tau}\right) \stackrel{d}{\rightarrow} f(\sigma W) \quad \text { in } \mathbb{R} \quad \text { as } \tau \rightarrow 0
$$

or, equivalently,

$$
\lim _{\tau \rightarrow 0} \nu\left(f\left(w_{\tau}\right) \leq x\right)=\mathbb{P}(f(\sigma W) \leq x)
$$

for all $x \in \mathbb{R}$ that are points of continuity of the right-hand side, i.e., such that $\mathbb{P}(f(\sigma W)=x)=0$. Condition (2.2) is the way that Donsker [5] expressed his FCLT and the convergence of the functionals explains the name. In particular, condition (2.2) is equivalent to convergence of one-dimensional distributions for $f=\pi_{t}$ and $t>0$, and to convergence of finite dimensional distributions for $f$ being a finite linear combination of projections, by the Cramer-Wald theorem. However, the result goes beyond convergence of finite dimensional distributions, since, for example, we can take $f(\psi)=\sup _{0 \leq s \leq t} \psi(s)$ and many others; see [3]. Some special functionals of random walks were treated by Erdös and Kac [6], who noted a principle that the limit depends only on the single parameter $\sigma$ and is the same for all random walks. Thus the FCLT is also called the (weak) invariance principle.

We next recall well-known results (see e.g. [13, Chapter 5]) about the solutions of one-dimensional stochastic differential equations. Let $A: \mathbb{R} \rightarrow \mathbb{R}$ be a locally Lipschitz function, i.e., for each $k$ there is a constant $L_{k}>0$ such that for every $\left|z_{1}\right|,\left|z_{2}\right| \leq k$ :

$$
\left|A\left(z_{1}\right)-A\left(z_{2}\right)\right| \leq L_{k}\left|z_{1}-z_{2}\right| .
$$

Consider the equation

$$
Z(t)=y_{0}+\int_{0}^{t} A(Z(s)) d s+\sigma W(t)
$$

where $z_{0} \in \mathbb{R}, \sigma>0$, and $W$ is the Wiener process on $(\Omega, \mathcal{F}, \mathbb{P})$. Then there exists a unique process $Z$ such that if $e=\inf \{t \geq 0: Z(t)=-\infty$ or $Z(t)=+\infty\}$, then $Z$ is continuous on $[0, e)$,

$$
\mathbb{P}\left(Z(t)=z_{0}+\int_{0}^{t} A(Z(s)) d s+\sigma W(t) \text { for all } t<e\right)=1,
$$

and $Z$ on the set $\{e<\infty\}$ stays after $e$ at $-\infty$ or $+\infty$ at which it exits the state space $(-\infty, \infty)$. To check whether $Z$ is non-exploding we can use Feller's test for explosions [8]. By [13, Theorem 5.5.29], we have $e=\infty$ a.s. if and only if

$$
\phi( \pm \infty)=\infty, \quad \text { where } \phi(x)=\int_{0}^{x} \int_{y}^{x} \exp \left\{-\frac{2}{\sigma^{2}} \int_{y}^{u} A(z) d z\right\} d u d y .
$$

In particular, if $A$ is globally Lipschitz, i.e., (2.3) holds with $k=\infty$, then $e=\infty$, thus $Z$ is nonexploding. Finally, let $f: \mathbb{R} \rightarrow(0, \infty)$ be continuous, strictly increasing, and invertible with $f(-\infty)=0$ and $f(+\infty)=\infty$. If the process $Z$ exits its state space at the time $e$ then the process $X=f(Z)$ exits its state space $(0, \infty)$ exactly at the same time and vice-versa.

\section{Perturbations from deterministic systems}

In this section we describe the case when $\xi_{j}=T^{j}, j \geq 0$, and $T$ is an ergodic measure preserving transformation (e.m.p.t.) on the probability space $(Y, \mathcal{B}, \nu)$, that is $\nu$ is invariant for $T$, i.e., $\nu\left(T^{-1}(B)\right)=$ $\nu(B)$ for all $B \in \mathcal{B}$ and $T$ is ergodic (with respect to $\nu$ ), i.e., for each $B \in \mathcal{B}$ with $T^{-1}(B)=B$ we have $\nu(B) \in\{0,1\}$. Let $L^{p}(\nu)=L^{p}(Y, \mathcal{B}, \nu)$ for $p \geq 1$. We define the transfer operator $\mathcal{P}_{T}: L^{1}(\nu) \rightarrow L^{1}(\nu)$ by

$$
\int_{B} \mathcal{P}_{T} h(y) \nu(d y)=\int_{T^{-1}(B)} h(y) \nu(d y), \quad h \in L^{1}(\nu), B \in \mathcal{B} .
$$


We have the following consequence of [20, Theorem 1] and the Birkhoff ergodic theorem.

Theorem 3.1. Let $T$ be an e.m.p.t. on $(Y, \mathcal{B}, \nu)$ and let $h \in L^{2}(\nu)$ be such that

$$
\int_{Y} h(y) \nu(d y)=0 \quad \text { and } \quad\|h\|_{2}^{2}=\int_{Y} h^{2}(y) \nu(d y)>0 .
$$

If there is $\beta>1 / 2$ such that

$$
\limsup _{n \rightarrow \infty} n^{\beta}\left\|\mathcal{P}_{T}^{n} h\right\|_{2}<\infty
$$

then condition (1.12) holds with $\sigma_{0}^{2}=\|h\|_{2}^{2}$ and the FCLT holds $w_{\tau} \stackrel{d}{\rightarrow} \sigma W$ with

$$
\sigma=\lim _{n \rightarrow \infty} \frac{\left\|\sum_{j \leq n} h \circ T^{j}\right\|_{2}}{\sqrt{n}} .
$$

We refer the reader to [14, Section 2] for a collection of various examples of transformations and functions $h$ for which (3.1) is satisfied. Observe that if $\mathcal{P}_{T}(h)=0$ then (3.1) holds and

$$
\int h(y) h\left(T^{j}(y)\right) \nu(d y)=\int \mathcal{P}_{T}^{j} h(y) h(y) \nu(d y)=0
$$

for all $j \geq 1$, which gives $\sigma^{2}=\sigma_{0}^{2}>0$. In particular, the variables $h\left(\xi_{j}\right)$ are uncorrelated and the same conclusion holds as in Corollary 1.3. We illustrate solvability of the equation $\mathcal{P}_{T}(h)=0$ with the tent map.

Example 3.2. The tent map on $[-1,1]$ defined by (1.3) is ergodic with respect to the normalized Lebesgue measure on $[-1,1]$ and has a transfer operator given by

$$
\mathcal{P}_{T} h(y)=\frac{1}{2}\left[h\left(\frac{1}{2} y-\frac{1}{2}\right)+h\left(\frac{1}{2}-\frac{1}{2} y\right)\right] .
$$

Note that we have $\mathcal{P}_{T} h=0$ iff $h$ is such that $h(z)+h(-z)=0$. In particular, let $r \in \mathbb{R}$ and define $h(y)=y^{r}$ for $y \geq 0$ and $h(y)=-h(-y)$ for $y<0$. Then $h \in L^{2}(\nu)$ iff $r>-1 / 2$.

Remark 3.3. If $\mathcal{P}_{T}(h) \neq 0$ then it might be difficult to calculate the exact value of $\sigma$ directly from formula (3.2) and it can also happen that $\sigma=0$, a situation which should be avoided to get a diffusion process in the limit. The following argument might be helpful [20, Corollary 3]. If $h$ is bounded and

$$
\limsup _{n \rightarrow \infty} n^{2 \beta}\left\|\mathcal{P}_{T}^{n} h\right\|_{1}<\infty
$$

for some $\beta>1 / 2$, then condition (3.1) holds, since $\left\|\mathcal{P}_{T}^{n} h\right\|_{2} \leq\|h\|_{\infty}^{1 / 2}\left\|\mathcal{P}_{T}^{n} h\right\|_{1}^{1 / 2}$. Moreover,

$$
\sigma^{2}-\sigma_{0}^{2}=2 \sum_{n=1}^{\infty} \int h(y) h\left(T^{n}(y)\right) \nu(d y)=2 \sum_{n=1}^{\infty} \int \mathcal{P}_{T}^{n} h(y) h(y) \nu(d y)
$$

and $\sigma=0$ if and only if $h=f \circ T-f$ for some $f \in L^{1}(\nu)$.

Finally, we give two particular examples of transformations for which $\sigma^{2} \neq \sigma_{0}^{2}$. 
Example 3.4. The dyadic map on $[-1,1]$ is given by

$$
T(y)=\left\{\begin{array}{l}
2 y+1, y \in[-1,0] \\
2 y-1, y \in(0,1]
\end{array}\right.
$$

Like the tent map, it is ergodic with respect to the normalized Lebesgue measure on $[-1,1]$. It has a transfer operator given by

$$
\mathcal{P}_{T} f(y)=\frac{1}{2}\left[h\left(\frac{1}{2} y-\frac{1}{2}\right)+h\left(\frac{1}{2} y+\frac{1}{2}\right)\right] .
$$

If we take $h(y)=y$ then $\mathcal{P}_{T} h=h / 2$ and, by iterating this equality, we obtain $\mathcal{P}_{T}^{n} h=h / 2^{n}$ for all $n$. Thus condition (3.2) holds. In this example we have $\sigma_{0}^{2}=1 / 3$ and, by Remark $3.3, \sigma^{2}-\sigma_{0}^{2}=2 \sigma_{0}^{2}$, hence $\sigma^{2}=1$. Consequently, the process $X$ will satisfy

$$
X(t)=x_{0}+\int_{0}^{t} X(s)\left(g(X(s))+\frac{1}{3}\right) d s+\int_{0}^{t} X(s) d W(s) .
$$

Example 3.5. The quadratic map

$$
T(y)=1-2 y^{2}
$$

on $[-1,1]$ is ergodic with respect to the measure $\nu$ on $[-1,1]$ with the density

$$
g_{*}(y)=\frac{1}{\pi \sqrt{1-y^{2}}} .
$$

The transfer operator is given by

$$
\mathcal{P}_{T} h(y)=\frac{1}{2}\left[h\left(\sqrt{\frac{1}{2} y+\frac{1}{2}}\right)+h\left(-\sqrt{\frac{1}{2} y+\frac{1}{2}}\right)\right] .
$$

Here we also have $\mathcal{P}_{T} h=0$ for $h(y)=y$ and $\sigma_{0}^{2}=\sigma^{2}=1 / 2$. If, instead, we take $h(y)=y^{2}-1 / 2$, then $\mathcal{P}_{T} h(y)=y / 2$ and $\mathcal{P}_{T}^{2} h=0$. Thus, for such $h$ we have $\sigma^{2}-\sigma_{0}^{2}=1 / 2$.

\section{Proofs}

In this section we first study integral equations

$$
z(t)=z_{0}+\int_{0}^{t} b(z(s)) d s+\psi(t), \quad t \geq 0
$$

for $\psi \in \mathbb{D}$, where $z_{0} \in \mathbb{R}$ is fixed and $b: \mathbb{R} \rightarrow \mathbb{R}$ is locally Lipschitz-continuous. The proofs of Theorems 1.1 and 1.2 will be given later on. We have the following results.

Theorem 4.1 ([17, Theorem 4.1]). Assume that $b: \mathbb{R} \rightarrow \mathbb{R}$ is globally Lipschitz-continuous. Then for each $\psi \in \mathbb{D}$ the integral equation (4.1) has a unique solution $z \in \mathbb{D}$ and if $\psi$ is a continuous function then so is $z$. The function $G: \mathbb{D} \rightarrow \mathbb{D}$ mapping each $\psi$ into the corresponding $z$ is measurable and continuous in $\mathbb{D}$. 
Theorem 4.2. For each $k>0$, let $S_{k}: \mathbb{D} \rightarrow[0, \infty]$ be defined by

$$
S_{k}(\psi)=\inf \{t \geq 0:|\psi(t)| \geq k \text { or }|\psi(t-)| \geq k\}, \quad \psi \in \mathbb{D},
$$

where we set $\inf \emptyset=+\infty$, and let the mapping $G_{k}: \mathbb{D} \rightarrow \mathbb{D}$ be given by the integral equation

$$
z(t)=z_{0}+\int_{0}^{t} b_{k}(z(s)) d s+\psi(t), \quad t \geq 0,
$$

where $b_{k}$ is a Lipschitz continuous function on $\mathbb{R}$ such that $b_{k}(z)=b(z)$ for $|z| \leq k$. Then, for each $\psi \in \mathbb{D}$, the following limits exist

$$
G_{\infty}(\psi)(t):=\lim _{k \uparrow \infty} G_{k}(\psi)(t) \quad \text { for all } t<S_{\infty}(\psi), \quad S_{\infty}(\psi):=\lim _{k \uparrow \infty} S_{k}\left(G_{k}(\psi)\right),
$$

and $G_{\infty}(\psi)$ satisfies (4.1) for $t \in\left[0, S_{\infty}(\psi)\right)$. The mapping $G: \mathbb{D} \rightarrow \mathbb{D}$ defined by

$$
G(\psi)(t)= \begin{cases}G_{\infty}(\psi)(t), & t<S_{\infty}(\psi) / 2 \\ G_{\infty}(\psi)\left(S_{\infty}(\psi) / 2\right), & t \geq S_{\infty}(\psi) / 2\end{cases}
$$

is measurable and $\mathbb{D}_{0}:=\left\{\psi \in \mathbb{D}: S_{\infty}(\psi)=\infty\right\} \in \mathcal{D}$.

Moreover, if $\psi_{n} \rightarrow \psi$ and $\psi \in \mathbb{D}_{0}$ then $G\left(\psi_{n}\right) \rightarrow G(\psi)$.

Proof. Let $l \geq k>\left|z_{0}\right|+|\psi(0-)|$. For $t<S_{k}\left(G_{k}(\psi)\right)$ we have $\sup _{s \leq t}\left|G_{k}(\psi)(s)\right|<k$, thus $\left.b_{k}\left(G_{k}(\psi)(s)\right)=b_{l}\left(G_{k}(\psi)(s)\right)\right)$ for all $s \leq t$ and

$$
\begin{aligned}
\left|G_{k}(\psi)(t)-G_{l}(\psi)(t)\right| & \leq \int_{0}^{t}\left|b_{l}\left(G_{k}(\psi)(s)\right)-b_{l}\left(G_{l}(\psi)(s)\right)\right| d s \\
& \leq L_{l} \int_{0}^{t}\left|G_{k}(\psi)(s)-G_{l}(\psi)(s)\right| d s
\end{aligned}
$$

where $L_{l}$ is the Lipschitz constant of $b_{l}$. From the Gronwall's inequality it follows that

$$
G_{k}(\psi)(t)=G_{l}(\psi)(t) \quad \text { for all } t<S_{k}\left(G_{k}(\psi)\right), k \leq l .
$$

Observe that, for each $\psi$, the function $k \rightarrow S_{k}(\psi)$ is nondecreasing and hence $S_{k}\left(G_{l}(\psi)\right) \leq S_{l}\left(G_{l}(\psi)\right)$ for $k \leq l$. Since $S_{k}\left(G_{k}(\psi)\right)=S_{k}\left(G_{l}(\psi)\right)$, the sequence $S_{k}\left(G_{k}(\psi)\right)$ is nondecreasing and the limit $S_{\infty}(\psi)$ exists. We have $G_{k}(\psi)(s)=\lim _{l \rightarrow \infty} G_{l}(\psi)(s)=G_{\infty}(\psi)(s)$ for all $s \leq t<S_{k}\left(G_{k}(\psi)\right)$. Hence, $G_{\infty}(\psi)$ satisfies (4.1) for $t<S_{k}\left(G_{k}(\psi)\right)$, and letting $k \rightarrow \infty$ we obtain that $G_{\infty}(\psi)$ satisfies (4.1) for $t<S_{\infty}(\psi)$. For each $k>0, S_{k}$ is measurable, by [12, Propositions VI.2.10], and $G_{k}$ is measurable, by Theorem 4.1, thus the composition $S_{k} \circ G_{k}$ is measurable and $S_{\infty}$, being the limit, is measurable. Hence $\mathbb{D}_{0} \in \mathcal{D}$. The mapping $G$ is measurable, since $\pi_{t} \circ G_{k}$ are measurable, where $\pi_{t}$ are projections, as well as the limit $\pi_{t} \circ G_{\infty}$, when restricted to the set $\left\{\psi: S_{\infty}(\psi)>2 t\right\} \in \mathcal{D}$. Finally, let $\psi \in \mathbb{D}_{0}$ and $\psi_{n} \rightarrow \psi$. Fix $m>0$. Since $S_{\infty}(\psi)=+\infty$, we can find $k$ such that $2 m<S_{k}\left(G_{k}(\psi)\right)$. Then $G(\psi)(s)=G_{k}(\psi)(s)$ for all $s \leq m$. Since $G_{k}$ is continuous, we have $G_{k}\left(\psi_{n}\right) \rightarrow G_{k}(\psi)$. It is easily seen that $\liminf _{n \rightarrow \infty} S_{k}\left(G_{k}\left(\psi_{n}\right)\right) \geq S_{k}\left(G_{k}(\psi)\right)$, thus $2 m<S_{k}\left(G_{k}\left(\psi_{n}\right)\right)$ for all sufficiently large $n$, and for such $n$ we have $G\left(\psi_{n}\right)(s)=G_{k}\left(\psi_{n}\right)(s)$ for all $s \leq m$. Hence, we conclude that

$$
\lim _{n \rightarrow \infty} \sup _{0 \leq s \leq m}\left|G\left(\psi_{n}\right)(s)-G(\psi)(s)\right|=0,
$$

which completes the proof.

Remark 4.3. If $S_{\infty}(\psi)<\infty$ then the function $G_{\infty}(\psi)$ is only well defined for $t \in\left[0, S_{\infty}(\psi)\right)$. We defined $G$ by stopping at the finite time $S_{\infty}(\psi) / 2$, but instead one can choose any measurable time $t_{\infty}(\psi)<S_{\infty}(\psi)$. Note also that if $\psi$ is continuous then $G(\psi)$ is continuous. 
Theorem 1.1 follows directly from the CMT and Theorem 4.2, since $A$, being of class $C^{1}$, is locally Lipschitz and the assumptions are such that $z_{\tau}, \sigma W \in \mathbb{D}_{0}$.

Proof of Theorem 1.2. For all sufficiently small $\tau$, define $z_{\tau}(t)=\log \left(x_{\tau}(t)\right), t \geq 0$. We have $z_{\tau}^{\prime}(t)=$ $g\left(\exp \left(z_{\tau}(t)\right)\right)$ if $j \tau<t<(j+1) \tau$ and

$$
z(j \tau)=\log \left(x_{\tau}(j \tau)\right)=\log \left(x_{\tau}(j \tau)\left(1+\sqrt{\tau} h\left(\xi_{j}\right)\right)\right)=z(j \tau-)+\log \left(1+\sqrt{\tau} h\left(\xi_{j}\right)\right) .
$$

Consequently, $z_{\tau}$ is the solution of the integral equation (4.1), where $b(z)=g(\exp (z)), z \in \mathbb{R}, z_{0}=\log x_{0}$, and $\psi(t)=W_{\tau}(t)$ with

$$
W_{\tau}(t)=\sum_{0 \leq j \tau \leq t} \log \left(1+\sqrt{\tau} h\left(\xi_{j}\right)\right) .
$$

We claim that conditions (1.6) and (1.12) imply $W_{\tau} \stackrel{d}{\rightarrow} W_{0}$, where

$$
W_{0}(t)=\sigma W(t)-\frac{1}{2} \sigma_{0}^{2} t, \quad t \geq 0
$$

Observe that, for $|a|<1$,

$$
\log (1+a)=\sum_{n=1}^{\infty} \frac{(-1)^{n+1}}{n} a^{n}
$$

and therefore

$$
\left|\log (1+a)-\left(a-\frac{1}{2} a^{2}\right)\right| \leq \sum_{n=3}^{\infty}|a|^{n} \leq \frac{|a|^{3}}{1-|a|} \leq 2|a|^{3},
$$

if $|a| \leq 1 / 2$. Thus,

$$
W_{\tau}(t)=w_{\tau}(t)-y(t)+R_{\tau}(t) \quad \text { with } y(t)=\frac{1}{2} \sigma_{0}^{2} t, \quad t \geq 0,
$$

where

$$
R_{\tau}(t)=y(t)-y_{\tau}(t)+r_{\tau}(t), \quad y_{\tau}(t)=\frac{1}{2} \tau \sum_{0 \leq j \tau \leq t} h^{2}\left(\xi_{j}\right), \quad t \geq 0,
$$

and the error term $r_{\tau}$ satisfies $\left|r_{\tau}(t)\right| \leq C \sqrt{\tau}(t+\tau)$ for some constant $C$, for all $t$, and all sufficiently small $\tau$, since $h$ is bounded. Note that the mapping $\psi \mapsto \psi-y$ is measurable and continuous, since $y$ is continuous. Thus $w_{\tau}-y \stackrel{d}{\rightarrow} W_{0}$. From assumption $(1.12)$ it follows that $y_{\tau}(t) \rightarrow y(t)$ a.s. for each $t$. Since, for each $\tau$, the process $y_{\tau}$ has nondecreasing paths and $y$ is continuous, we obtain $\sup _{0 \leq s \leq m} \mid y_{\tau}(s)-$ $y(s) \mid \rightarrow 0$ a.s. as $\tau \rightarrow 0$ for all $m$. Consequently, $\sup _{0 \leq s \leq m}\left|R_{\tau}(s)\right| \rightarrow 0$ a.s. as $\tau \rightarrow 0$ for all $m$, which implies the claim (see [3, Sections 2 and 6$]$ ).

By assumption $W_{\tau} \in \mathbb{D}_{0}$ and $z_{\tau}=G\left(W_{\tau}\right)$. From Itô formula and the final remark at the end of section 2 , it follows that $\zeta=\infty$ a.s. and $X$ is the unique solution of (1.13) iff $S_{\infty}\left(W_{0}\right)=\infty$ a.s. and $Z=\log X$ is the unique solution of

$$
Z(t)=z_{0}+\int_{0}^{t}\left(b(Z(s))-\frac{\sigma_{0}^{2}}{2}\right) d s+\sigma W(t) .
$$

Thus $W_{0} \in \mathbb{D}_{0}$ and $Z=G\left(W_{0}\right)$ a.s. From Theorem 4.2 and the CMT it follows that $G\left(W_{\tau}\right) \stackrel{d}{\rightarrow} G\left(W_{0}\right)$. The mapping $H: \mathbb{D} \rightarrow \mathbb{D}$ defined by $H(\psi)(t)=\exp (\psi(t)), t \geq 0$, is continuous and measurable. Hence $H\left(z_{\tau}\right) \stackrel{d}{\rightarrow} H(Z)$ and the process $X(t)=H(Z)(t), t \geq 0$, is the unique solution of (1.13).

Remark 4.4. The assumption that each solution of $x^{\prime}(t)=x(t) g(x(t)), x(0)=x_{0}$, does not exit the state space $(0, \infty)$ can be relaxed. Then we need to define $x_{\tau}$ accordingly. We can take $x_{\tau}(t)=\log \left(z_{\tau}(t)\right)$ and $z_{\tau}=G\left(W_{\tau}\right)$, where $G$ is as in Theorem 4.2, which means that $x_{\tau}$ satisfies (1.11) for $t \in\left[0, t_{\tau} / 2\right]$ where $t_{\tau}=\inf \left\{t \geq 0: x_{\tau}(t) \notin(0, \infty)\right.$ or $\left.x_{\tau}(t-) \notin(0, \infty)\right\}$ and $x_{\tau}(t)=x_{\tau}\left(t_{\tau}\right)$ for $t \geq t_{\tau} / 2$. Then $x_{\tau} \stackrel{d}{\rightarrow} X$ provided that $X$ is non-exploding. Similarly, we can redefine the Langevin equation in Theorem 1.1. 


\section{Concluding remarks}

In this paper we show that we can easily derive convergence in distribution of non-Markov piecewise continuous deterministic processes to diffusion processes from the FCLT and the continuous mapping theorem. We can extend our results for Langevin-type equations in Theorem 1.1 to situations when $w_{\tau}$ converges to a discontinuous limit, as in the case of Lévy walks, but we need to use the Skorohod $J_{1}$-topology in $\mathbb{D}$. This may occur when $h \notin L^{2}(\nu)$ and $\kappa(\tau)=\tau^{1 / \alpha}$ with $\alpha \in(0,2)$ in (1.1) with the change of $\sqrt{\tau}$ to $\tau^{1 / \alpha}$ in the definition (1.6) of $w_{\tau}$. Then we may have convergence in distribution to Lévy $\alpha$-stable processes (also known as Lévy flights), see [21,22] and the references therein. We give one example based on the tent map. If $h$ is as in Example 3.2 with $r=-1 / \alpha$ and $\alpha \in(0,2)$ then $h$ has infinite variance and power law distribution. From [22, Section 4.2] it follows that there exists

a positive constant $\sigma_{\alpha}$ such that $w_{\tau} \stackrel{d}{\rightarrow} \sigma_{\alpha} W_{\alpha}$, where $W_{\alpha}$ is a symmetric Lévy $\alpha$-stable process, i.e., $W_{\alpha}(0)=0, W_{\alpha}$ has stationary independent increments, and $W_{\alpha}(1)$ has the characteristic function of the form $E\left(\exp \left\{i u W_{\alpha}(1)\right\}\right)=\exp \left\{-c_{\alpha}|u|^{\alpha}\right\}, u \in \mathbb{R}$, where $c_{\alpha}$ is a positive constant and $\alpha \in(0,2)$. Note that the case of $\alpha=2$ corresponds to a constant multiple of the Wiener process. Since the mapping in Theorem 4.1 is continuous in the Skorohod $J_{1}$-topology by [17, Theorem 4.1], one can show that the mapping $G$ in Theorem 4.2 is also continuous in that topology, thus Theorems 1.1 and 4.2 remain true for the Skorohod $J_{1}$-topology. They also remain true in the case when all processes have values in $\mathbb{R}^{d}$.

It is not so straightforward to extend Theorem 1.2 to the general case of multiplicative noise leading to equations of the form

$$
X(t)=x_{0}+\int_{0}^{t}\left(g(X(s))+\frac{\sigma^{2}-\sigma_{0}^{2}}{2} a^{\prime}(X(s))\right) a(X(s)) d s+\sigma \int_{0}^{t} a(X(s)) d W(s),
$$

where the function $a$ is sufficiently smooth. We will report on that in a separate communication. However, our results, even in the simple case of $a(x)=x, x>0$, show that if the noise in the growth rate is bounded and uncorrelated then in the limit we always obtain the Itô equation. For correlated noise we can get an equation which is neither Itô nor Stratonovich. In particular, a question [4] whether one should use Itô or Stratonovich calculus is irrelevant as long as stochastic equations are not written ad hoc. Deriving them as approximations should give the correct answer.

Acknowledgements. This work was supported by State Committee for Scientific Research Grant N N201 608240.

\section{References}

[1] C. Beck. Ergodic properties of a kicked damped particle. Commun. Math. Phys., 130 (1990), 51-60.

[2] C. Beck, G. Roepstorff. From dynamical systems to the Langevin equation. Phys. A, 145 (1987), 1-14.

[3] P. Billingsley. Convergence of probability measures, 2nd edition. John Wiley \& Sons Inc., New York, 1999.

[4] C. A. Braumann. Itô versus Stratonovich calculus in random population growth. Math. Biosci., 206 (2007), 81-107.

[5] M. D. Donsker. An invariance principle for certain probability limit theorems. Mem. Amer. Math. Soc., 1951 (1951), 12.

[6] P. Erdös, M. Kac. On certain limit theorems of the theory of probability. Bull. Amer. Math. Soc., 52 (1946), $292-302$.

[7] S. N. Ethier, T. G. Kurtz. Markov processes. Characterization and convergence. John Wiley \& Sons Inc., New York, 1986.

[8] W. Feller. The parabolic differential equations and the associated semi-groups of transformations. Ann. of Math., (2) 55 (1952), 468-519.

[9] D. Givon, R. Kupferman. White noise limits for discrete dynamical systems driven by fast deterministic dynamics. Phys. A 335 (2004), 385-412.

[10] G. A. Gottwald, I. Melbourne. Homogenization for deterministic maps and multiplicative noise. Proc. R. Soc. A 469 (2013), 20130201.

[11] N. Ikeda, S. Watanabe. Stochastic differential equations and diffusion processes, 2nd edition. North-Holland Publishing Co., Amsterdam, 1989.

[12] J. Jacod, A. N. Shiryaev. Limit theorems for stochastic processes, 2nd edition. Springer-Verlag, Berlin, 2003.

[13] I. Karatzas, S. E. Shreve. Brownian motion and stochastic calculus, 2nd edition. Springer-Verlag, New York, 1991.

[14] M. C. Mackey, M. Tyran-Kamińska. Deterministic Brownian motion: The effects of perturbing a dynamical system by a chaotic semi-dynamical system. Phys. Rep., 422 (2006), 167-222. 
[15] I. Melbourne, A. M. Stuart. A note on diffusion limits of chaotic skew-product flows. Nonlinearity 24 (2011), $1361-1367$.

[16] F. Merlevède, M. Peligrad, S. Utev. Recent advances in invariance principles for stationary sequences. Probab. Surv., 3 (2006), 1-36.

[17] G. Pang, R. Talreja, W. Whitt. Martingale proofs of many-server heavy-traffic limits for Markovian queues. Probab. Surv. 4 (2007), 193-267.

[18] A. V. Skorohod. Limit theorems for stochastic processes. Teor. Veroyatnost. i Primenen., 1 (1956), $289-319$.

[19] D. W. Stroock, S. R. S. Varadhan. Multidimensional diffusion processes. Springer-Verlag, Berlin, 1979.

[20] M. Tyran-Kamińska. An invariance principle for maps with polynomial decay of correlations. Comm. Math. Phys., 260 (2005), 1-15.

[21] M. Tyran-Kamińska. Convergence to Lévy stable processes under some weak dependence conditions. Stochastic Process. Appl., 120 (2010), 1629-1650.

[22] M. Tyran-Kamińska. Weak convergence to Lévy stable processes in dynamical systems. Stoch. Dyn., 10 (2010), 263-289.

[23] W. Whitt. Some useful functions for functional limit theorems. Math. Oper. Res., 5 (1980), 67-85.

[24] W. Whitt. Stochastic-process limits. Springer-Verlag, New York, 2002.

[25] N. Wiener. The differential space. J. Math. Phys., 2 (1923), 121-174.

[26] E. Wong, M. Zakai. On the convergence of ordinary integrals to stochastic integrals. Ann. Math. Statist., 36 (1965), $1560-1564$. 